\title{
Evidence for the presence of synovial sheaths surrounding the extensor tendons at the metacarpophalangeal joints
}

\author{
Yousra J. Dakkak ${ }^{1}$, Friso P. Jansen ${ }^{2}$, Lambertus J. Wisse ${ }^{2}$, Monique Reijnierse ${ }^{3}$, Annette H.M. van der \\ Helm-van Mil' ${ }^{1}$, Marco C. DeRuiter ${ }^{2}$ \\ ${ }^{1}$ Department of Rheumatology, Leiden University Medical Center, Leiden, The Netherlands \\ ${ }^{2}$ Department of Anatomy \& Embryology, Leiden University Medical Center, Leiden, The Netherlands \\ ${ }^{3}$ Department of Radiology, Leiden University Medical Center, Leiden, The Netherlands
}

\section{Corresponding author:}

Prof. Dr. A.H.M. van der Helm-van Mil, Dept. of Rheumatology, Leiden University Medical Center

P.O. Box 9600, 2300 RC Leiden, the Netherlands, Tel.: +31 71 5265655, Fax: +31 715266752

Email: A.H.M.van der Helm@lumc.nl

Keywords: tendon sheath, metacarpophalangeal, rheumatoid arthritis, tenosynovitis, peritendinitis, anatomy

\section{Abstract}

Background. MRI-detected inflammation around the metacarpophalangeal (MCP-)joints is prevalent in RA and poses a markedly increased risk of RA-development when present in arthralgia patients. Such inflammation is called 'peritendinitis', since anatomy literature reports no presence of a tenosynovial sheath at these tendons. However, the presence or absence of a synovial sheath at these extensor tendons has never been studied.

Methods. A macroscopy and microscopy study of extensor-tendons at the MCP-joints of two embalmed human hands was performed. Routine histology was performed with Haematoxylin-Eosin staining.

Results. We found evidence for the presence of synovial lining around extensor-tendons at MCPjoints. A delimited space surrounding the extensor digitorum tendon was observed, which was lined with an epithelium representing fibroblast-like synoviocytes.

Conclusion. Contrast-enhancement around extensor tendons at MCP joints observed on MRI in RA represents tenosynovitis and thus inflammation of synovial tissue. Further studies with larger numbers of specimen are warranted to study anatomic variants. In addition, the molecular composition of the tenosynovium remains to be characterized. 


\section{Introduction}

Human anatomy is one of the oldest cornerstones of medical education. It may seem surprising that advances in this field are still made. However, modern imaging techniques, such as MRI, visualize the human body in increasingly finer detail, posing new questions how to anatomically interpret what is seen.[1] For Rheumatoid Arthritis (RA) this applies to tenosynovitis of hand and foot, i.e. inflammation of the synovial sheaths that surround tendons, which was recently reported to be characteristic for RA.[2]

In the foot, MRI-detected inflammation surrounding metatarsophalangeal(MTP)-tendons occurs frequently in RA. Surprisingly, anatomic literature left it undetermined whether these tendons are surrounded by synovial sheaths. A recent anatomic study revealed that these tendons do possess synovial sheaths, both at flexor and extensor sides. This implies that MRI-detected inflammation surrounding MTP-tendons in RA represents tenosynovitis.[3]

In the hand, MRI-detected tenosynovitis is also prevalent in RA at the metacarpophalangeal(MCP)joints, including flexor and extensor tendons. Moreover, in patients with arthralgia, contrastenhancement around MCP extensor tendons poses a high risk of development of RA.[4] According to anatomic atlases, the extensor-tendons at MCP-joints lack a synovial sheath, therefore inflammation around extensor-tendons is termed 'peritendinitis'. The source of these anatomic images is unclear; to the best of our knowledge the presence or absence of a synovial sheath at these extensor tendons has not been studied. We hypothesized that the tissue surrounding the extensor-tendons of MCP-joints is not different from MTP-joints, and thus that a tenosynovial sheath is present.

Therefore, to reconsider the current anatomic information, and with the ultimate aim to improve our understanding of tissues inflamed in early RA, we performed a microscopy study of extensortendons at the MCP-joints. 


\section{Methods}

The study was conducted on two formalin-phenol embalmed hands. The specimens were obtained from bodies that were donated according to the Dutch Burial and Cremation Act to the department of Anatomy and Embryology at the Leiden University Medical Center for use in scientific research and medical education. All donors provided written informed consent during life.

The studies were performed at the Anatomy \& Embryology lab of the Leiden University Medical Center in Leiden, The Netherlands. The cutis and subcutis of the embalmed hands were removed. Blocks containing the extensor tendons located at the MCP-joint including surrounding connective tissue and part of the underlying proximal phalanx were removed from the following locations: MCP 1, 2 and 4 from patient 1, and MCP 3 from patient 2. Harvested tissues were embedded in paraffin. The paraffin blocks were transversely sliced in sections of $10 \mu \mathrm{m}$. The morphology of all tissues was examined by a haematoxylin and eosin (HE) staining and interpreted by two anatomists.

\section{Results}

Surrounding the extensor tendons a completely delimited space was noticed (Figure 1A). This space was lined with an epithelium, representing fibroblast-like synoviocytes which was 2 to 3 cellular layers thick (Figure 1B) and at places thickened including blood vessels (Figure 1C). The results of the extensor tendons at the second MCP-joint are presented, the other MCP-joints revealed a similar image. 
Figure 1. A-C) Histologic evaluation of a transverse section through tendon (T) and ossa digitorum, proximal phalanx (O) near MCP 2. B and C are enlargements indicated by the boxes in $\mathbf{A}$. Note the characteristic synovial layer (arrows) surrounding the space (S) around the tendon, representing fibroblast-like synoviocytes. Magnification: A: Bar = 1000 um; B: Bar = 100 um; C: Bar = 20 um. D) Axial T1 weighted fat suppressed image with gadolinium contrast (T1FS Gd) of the left hand shows extensor tenosynovitis at MCP 3 (arrow). At MCP 4 synovitis is present. E) Axial T1FS Gd image of the right hand of a different patient shows extensor tenosynovitis at MCP 4 and flexor tenosynovitis at MCP 3 (arrows from left to right respectively), illustrating the oval shape of the flexor tenosynovitis and the more flattened shape of the extensor tenosynovitis. Note the absence of synovitis at MCP 4 .
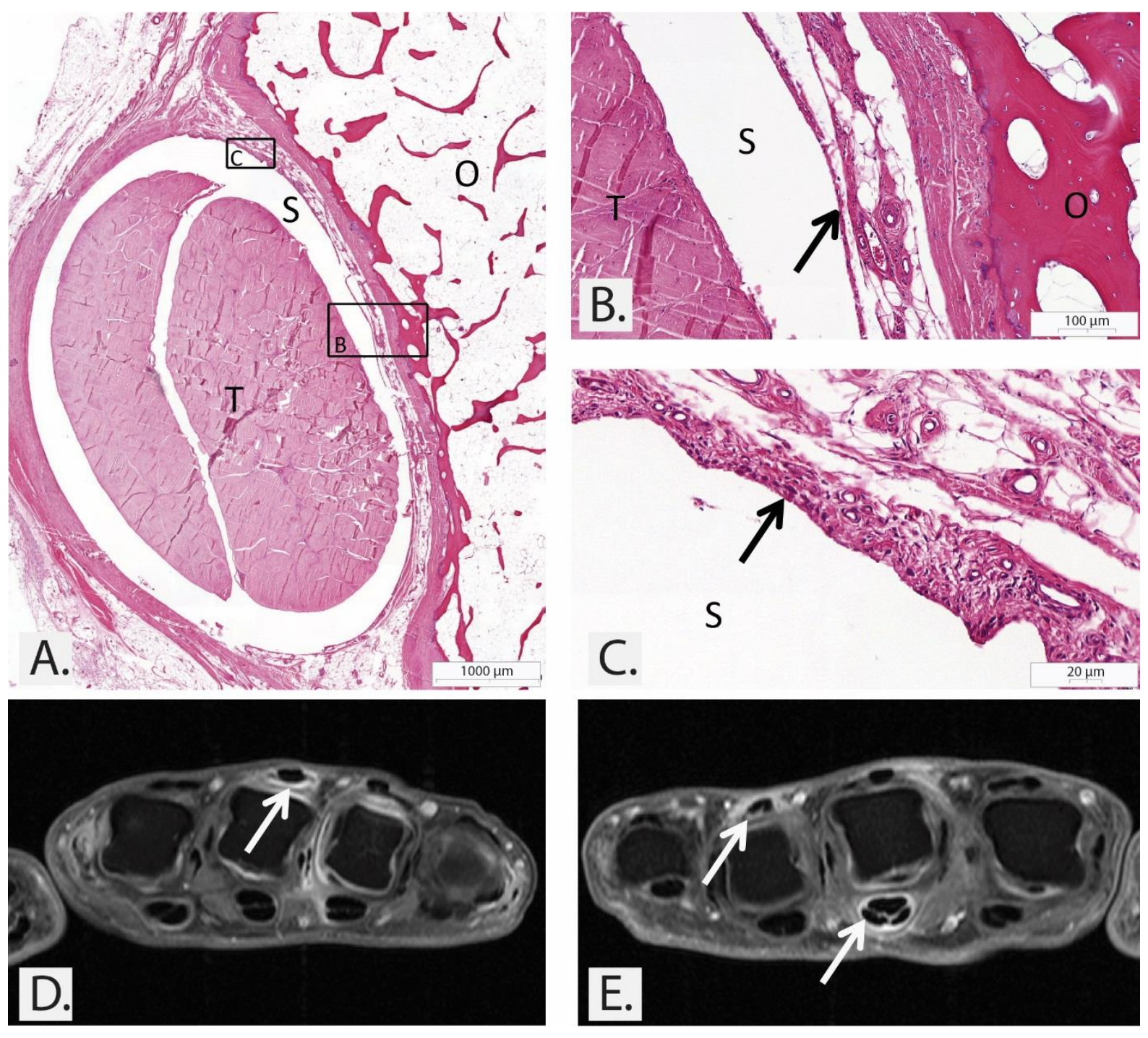


\section{Discussion}

We found evidence for the presence of synovial lining around extensor-tendons at MCP-joints, suggesting that contrast-enhancement around extensor tendons observed in RA represents tenosynovitis and thus inflammation of synovial tissue. An anatomical study of extensor-tendon sheaths from patients with active RA would be the ultimate proof, but it is challenging to obtain these materials. Interestingly, MRI-detected contrast-enhancement around MCP-extensor tendons is more flattened than around flexor-tendons (Figure 1D and 1E). This may be explained by fibrous bands that interconnect the extensor-tendons to prevent subluxation whilst flexing fingers, causing pressure on the tendon-sheaths (Supplementary File; Figure S1, Table S1 and S2). In addition, extensor-tendons are anatomically smaller than flexor-tendons, become flattened at the level of the MCP joints where together with the dorsal expansion of lumbrical and interosseous muscles, a so called extensor hood, is formed.[5, 6] Further studies, with larger numbers of specimen are warranted to study anatomic variants in tendon-sheaths, and using immunohistochemistry to further characterize the tenosynovium. Nonetheless, our data suggest that contrast-enhancement around extensor-tendons in RA represent tenosynovitis. This eventually may warrant modification of the classic anatomic pictures of the hand. Thus, advanced imaging led to multidisciplinary research that challenged previous assumptions and revisited anatomic knowledge, resulting in a better understanding of tissues that are inflamed in classified RA and early phases of RA-development. Ultimately this knowledge contributes to the unravelling of the pathophysiology of RA. 


\section{Acknowledgements}

\section{INFORMED CONSENT}

All donors provided written informed consent during life.

\section{ETHICS APPROVAL}

All anatomical specimen were obtained from human bodies that were donated according to the Dutch Burial and Cremation Act to the department of Anatomy and Embryology at the Leiden University Medical Center for use in scientific research and medical education. Since the bodies had been donated for medical research under this act, no additional local ethics approval was needed for this specific research. During life all the donors have signed an informed consent form to state that their body can be used for any kind of medical research, which was approved by the mayor of the city.

\section{AUTHOR CONTRIBUTIONS}

YJD, FPJ, LJW, MR, AHMvdHvM and MdR contributed to the conception and study design.

YJD, FPJ, LWW and MdR analysed the data.

YJD, FPJ, LWW, MR, AHMvdHvM and MdR contributed to interpretation of the data.

FPJ, LJW and MdR contributed to acquisition of the data.

YJD and AHMvdHvM wrote the first version of the manuscript and FPJ, LWW, MR and MdR revised it critically.

All authors read and approved the final version of the document. 


\section{FUNDING}

This work was supported by the European Research Council (ERC) under the European Union's Horizon 2020 research and innovation programme (Starting grant, agreement No 714312), and the Dutch Arthritis Society. The authors or their institutions did not receive any payment or services from another third party for any aspect of this work.

\section{COMPETING INTERESTS}

None declared.

\section{DATA SHARING STATEMENT}

Data can be requested from the corresponding author. 


\section{References}

1. Eizenberg, N., Anatomy and its impact on medicine: Will it continue? Australas Med J, 2015. 8(12): p. 373-7.

2. Rogier, C., S. Hayer, and A. van der Helm-van Mil, Not only synovitis but also tenosynovitis needs to be considered: why it is time to update textbook images of rheumatoid arthritis. Ann Rheum Dis, 2019.

3. Dakkak, Y.J., et al., Rheumatoid Arthritis and Tenosynovitis at the Metatarsophalangeal Joints: An Anatomic and MRI Study of the Forefoot Tendon Sheaths. Radiology, 2020: p. 191725.

4. Niemantsverdriet, E. and A.H.M. van der Helm-van Mil, Imaging detected tenosynovitis of metacarpophalangeal and wrist joints: an increasingly recognised characteristic of rheumatoid arthritis. Clin Exp Rheumatol, 2018. 36 Suppl 114(5): p. 131-138.

5. Dass, P., et al., A comprehensive study of the extensor tendons to the medial four digits of the hand. Chang Gung Med J, 2011. 34(6): p. 612-9.

6. Benjamin, M., E. Kaiser, and S. Milz, Structure-function relationships in tendons: a review. J Anat, 2008. 212(3): p. 211-28. 